\title{
Short-Term Wind Speed Forecasting Model based on Local Comparison and Mean Circular Tube
}

\author{
Xuezong Bai, Zongwen An*, Yunfeng Hou, and Jianxiong Gao \\ School of Mechanical and Electronical Engineering, Lanzhou University of Technology, Lanzhou, 730050, China
}

\begin{abstract}
It is significant to forecast the short-term wind speed for the safety of wind turbine blades and the optimization of power grid dispatching. Firstly, the local comparison method is established to forecast the mean wind speed. Secondly, the universal generating function (UGF) is used to express the wind speed as a multi-state random variable, state probability allocation and the state probability matrix are used to obtain the risk state probability, and equal dimension filling is used to update the information. Then, the maximum wind speed is calculated based on the mean wind speed and risk state probability. Thirdly, local comparison is used for error forecasting, and the forecasting errors are used to correct the forecasting wind speeds. Finally, the mean circular tube is constructed, and the mean wind speed, maximum wind speed, risk state probability, and average relative error are displayed in the combined mean circular tube together.
\end{abstract}

Keywords: short-term wind speed forecasting; local comparison; mean circular tube; state probability allocation; equal dimension filling; error forecasting

(Submitted on March 26, 2019; Revised on April 23, 2019; Accepted on June 15, 2019)

(C) 2019 Totem Publisher, Inc. All rights reserved.

\section{Introduction}

The wind turbine blade is the key component of wind turbines. It captures wind energy and transforms it into mechanical energy. Thus, the reliable operation of wind turbine blades is directly related to the safety and reliability of wind turbines [1]. Wind turbine blades are subjected to random wind loads continuously, and their performance is obviously affected by wind speed. If future wind speeds cannot be forecasted, the performance of wind turbine blades in the future is unknown. Therefore, the real-time and accurate monitoring and forecasting of wind speed is of great significance for analyzing the load of wind turbine blades and developing the prognostic and health management (PHM) of wind turbine blades [2]. Due to the strong volatility and randomness of wind speed, large-scale wind power will bring serious challenges to the security and stability of power grids. Wind speed forecasting is effective for power grids [3].

The accuracy of short-term wind speed forecasting is currently not ideal, and the average forecasting error is about $17 \%$ [4]. It is unnecessary to forecast the wind speed each second because a certain period of time is needed for the safety evaluation and operation control of wind turbine blades. It is difficult to complete wind speed forecasting, safety assessment, operation management decision, and decision implementation in a few seconds. Meanwhile, it also is difficult to forecast the wind speed after a few seconds accurately. Thus, it is reasonable to carry out ten-minute wind speed forecasting based on the ten-minute wind speed records provided by the wind speed monitors of the wind turbines. Therefore, in this paper, the period of short-term wind speed forecasting is determined to be ten minutes [5].

Wind speed forecasting methods can be generally divided into historical data-based forecasting methods and numerical weather forecasting data-based forecasting methods. The persistence method, time series method, neural network method, support vector machine method, and Kalman filter method are historical data based forecasting methods. In the persistence method, the state value at one time is taken as the forecasting value at the next time. This is a simple and efficient method, but changes in the random wind speed are not reflected [6]. In the time series method, the future state is forecasted based on past trends, and its premise is that time must be continuous. The response to state mutation is lagging behind, and large

\footnotetext{
* Corresponding author.

E-mail address: anzongwen@163.com
} 
amounts of data are needed for model fitting [7]. In the neural network method and support vector machine method, the wind speed is forecasted based on the law of numerical change, but the selection difficulty and over-fitting of model are inevitable [8-9]. In Kalman filter method, the wind speed is forecasted by using the state equation of linear system and a small amount of historical data, but it is difficult to establish the state equation and the measurement equation [10].

Because of the dimension, geomorphology, and solar radiation cycle, the change of wind speed is always periodic [11]. The load of the wind turbine blade under periodic wind speeds is also periodic. For example, the measured wind speed in a region of Inner Mongolia of China over the past three years is shown in Figure 1. The wind speed increases gradually from February to May, decreases gradually from May to August, rises again from August to October, falls again from October to January of the following year, and repeats the above changes since February. This shows the periodicity.

Intuitively, wind speeds seem to be irregular, but in fact, any wind speed data fragment is extremely similar to a fragment in the historical data, and some of the future states are also very similar. For example, a set of ten-minute wind speed records in the same region of Inner Mongolia are shown in Figure 2. Two groups of similar wind speeds are marked in red and blue, and the future values of each group are also very similar. There are still some other similar fragments that have not been marked out. This shows the repeatability [12].

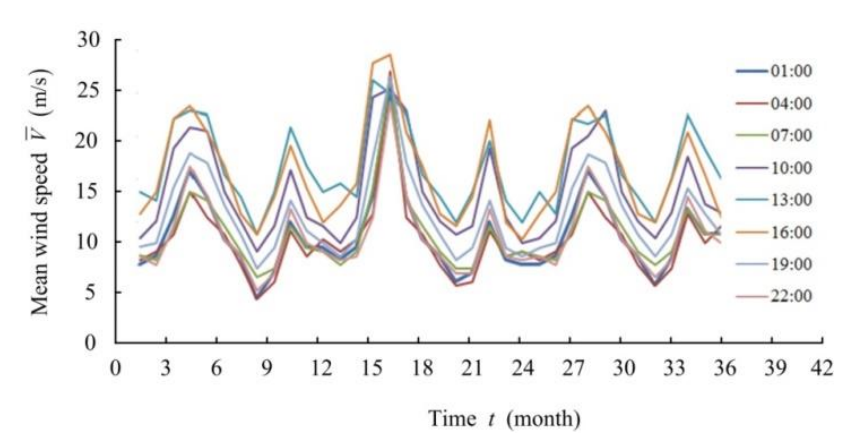

Figure 1. Periodicity of wind speed

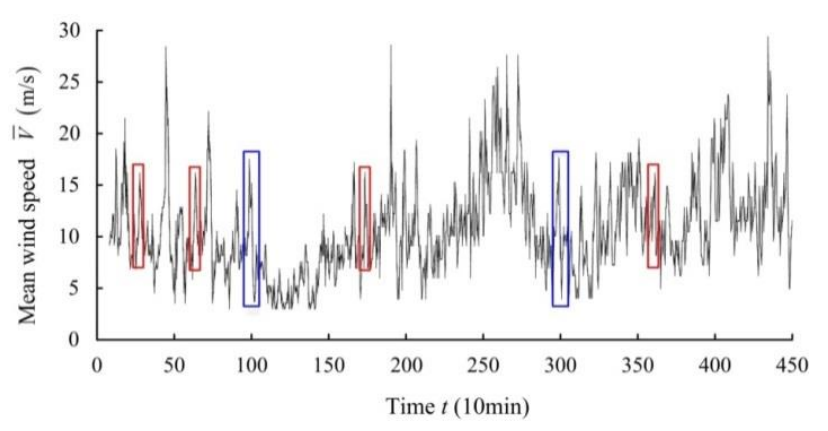

Figure 2. Repeatability of wind speed

Although sudden changes in wind speed appear irregular, they actually have certain patterns. From Figure 2, the sudden changes in wind speed always follow a number of gradual state changes, in other words, there is a preparation process. This shows the trends [13]. Based on the periodicity, repeatability, and trends, the future wind speed is forecasted by local comparison in Section 2.

\section{Local Comparison Method and Mean Wind Speed Forecasting}

As shown in Figure 3, a state data fragment is selected as a comparison fragment, and it is compared with other fragments starting with the first historical data. Then, several target fragments similar to the comparison fragment are obtained, such as target fragments 1 and 2. The differences between the comparison fragment and target fragments are obtained point by point. The maximum difference between the comparison fragment and target fragment 1 is $\delta_{1}$, and the maximum difference between the comparison fragment and target fragment 2 is $\delta_{2}$. In other words, target fragment 1 is similar to comparison fragment under permissible error $\delta_{1}$, and target fragment 2 is similar to comparison fragment under permissible error $\delta_{2}$. Because $\delta_{1}$ is less than $\delta_{2}$, target fragment 1 is more similar to the comparison fragment than target fragment 2. According to the trends of wind speed, if two fragments are similar, the value of the target fragment at the next time can be taken as the predicted value of the comparison fragment, and the predicted value is under a certain permissible error.

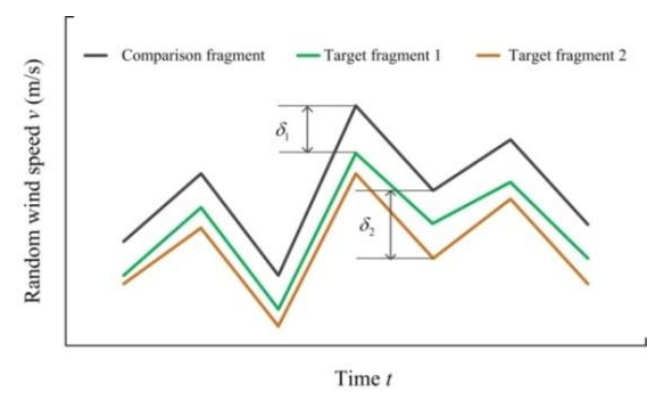

Figure 3. Schematic diagram of local comparison fragment 
According to the above idea, a set of permissible errors $\delta \in\left\{\delta_{1}, \delta_{2}, \cdots, \delta_{l} ; \delta_{1}<\delta_{2}<\cdots<\delta_{l}\right\}$ is given, and then the target fragments similar to the comparison fragments under the permissible error $\delta_{i}(i=1,2, \cdots, l)$ can be found from historical data. For example, based on the wind speed in Figure 2, the comparison fragment is selected as $C \in\{3,3,4,3.7\}$, the permissible errors are given as $\delta \in\{0.1,0.2,0.3,0.4,0.5\}$, and the target fragments under every permissible error are shown in Table 1. Evidently, the forecasting result is more accurate when the permissible error is small (such as $\delta=0.3$ ). When the permissible error is larger, the forecasting accuracy is lower, because the similar fragment would be found more quickly and a better fragment could be missed. However, the permissible error should not be too small, because it would be difficult or even impossible to find the target segment. The target fragment mentioned here is the target segment that meets the permissible error firstly.

Table 1. Comparison results under a series of permissible errors

\begin{tabular}{|c|c|c|c|c|}
\hline Permissible error $\delta$ & Target fragment $C$ & Forecasting value $v_{f}$ & Measured values $v_{a}$ & Forecasting error $\delta_{f}$ \\
\hline 0.1 & - & - & - & - \\
\hline 0.2 & $\{3,3,3.8,4.1\}$ & 3.4 & 4 & 0.6 \\
\hline 0.3 & $\{3,3,4.2,3.7\}$ & 4 & 4 & 0 \\
\hline 0.4 & $\{3,3,3.7,4\}$ & 3.7 & 4 & 0.3 \\
\hline 0.5 & $\{3,3,3.7,4\}$ & 3.7 & 4 & 0.3 \\
\hline
\end{tabular}

The permissible error is given as $\delta=0.3$, and the target fragments under a series of comparison lengths are shown in Table 2. The target fragment mentioned here is the target segment that meets the permissible error firstly. Obviously, the forecasting result is more accurate when the comparison length is long (such as $L=4$ ), because the similar fragment may be found more quickly and a better fragment must be missed. However, the comparison length should not be too long, because it is difficult or impossible to find the target segment.

Table 2. Comparison results under a series of comparison length

\begin{tabular}{|c|c|c|c|c|c|}
\hline $\begin{array}{c}\text { Comparison length } \\
L\end{array}$ & $\begin{array}{c}\text { Comparison fragment } \\
\delta\end{array}$ & $\begin{array}{c}\text { Target fragment } \\
C\end{array}$ & $\begin{array}{c}\text { Forecasting value } \\
v_{f}\end{array}$ & $\begin{array}{c}\text { Measured values } \\
v_{a}\end{array}$ & $\begin{array}{c}\text { Forecasting error } \\
\delta_{f}\end{array}$ \\
\hline 2 & $\{3,3\}$ & $\{3,3\}$ & 2.3 & 4 & 1.7 \\
\hline 3 & $\{3,3,4\}$ & $\{3,3.3,3.7\}$ & 2.7 & 3.7 & 1.0 \\
\hline 4 & $\{3,3,4,3.7\}$ & $\{3,3,4.2,3.7\}$ & 4 & 4 & 0 \\
\hline 5 & $\{3,3,4,3.7,4\}$ & $\{3,3.3,4,3.7,4.3\}$ & 3.3 & 2.7 & 0.6 \\
\hline 6 & $\{3,3,4,3.7,4,2.7\}$ & $\{3,3.2,4.2,4,4.1,2.4\}$ & 2.1 & 3.9 & 1.8 \\
\hline 7 & $\{3,3,4,3.7,4,2.7,3.8\}$ & - & - & - & - \\
\hline
\end{tabular}

In addition, the forecasting accuracy is also affected by the sample size of historical data. Wind change information should be included in the historical data sample. If the sample size is too small, the information is incomplete and it is difficult for the best target fragment to be found. Conversely, if it is too large, the comparison time will be extended and the efficiency will be reduced. In summary, the permissible error, comparison length, and sample size of historical data are three basic parameters that affect the forecasting accuracy of local comparison.

To solve the above mentioned problem, the target fragment should be searched by choosing the minimum permissible error and the longest comparison length at first. If the target fragment is found, the comparison will be stopped, or the comparison will be carried out by choosing a larger permissible error and a shorter comparison length. The comparison will continue until the target fragment is obtained. Considering the seasonality of the wind speed and the adequacy of the historical data, the wind speed data of one and a half years are taken as data samples, and the period is from October of the first year to March of the third year. There are slight differences in different regions. The flowchart of local comparison method is shown in Figure 4, and the main steps are as follows:

Step $1 l$ grades for permissible error and $k$ grades for comparison length are given. In this case, $l=5$ and $k=6$.

Step 2 The comparison length is equal to $k$, the permissible error is equal to $\delta_{1}$, and the target fragment is searched by comparing the comparison fragment with the historical data one by one. A target fragment will be chosen if all the data of the fragment meet the permissible error. If no target fragment is found, the permissible is equal to $\delta_{2}, \cdots, \delta_{l}$, and the target fragment is searched again from the historical data. 
Step 3 If the target fragment is still not found, the comparison is equal to $k-1$, the permissible error is equal to $\delta_{1}$, $\delta_{2}, \cdots, \delta_{l}$, and the target fragment will be searched again from the historical data in the same manner.

According to the local comparison method shown in Figure 4, based on the wind speed data of one and a half years from October 2013 to March 2014, 100 mean wind speeds and forecasting errors are shown in Figure 5. The forecasting error is between -2.5 and 2.7. The relative error is less than $13 \%$, which is lower than $17 \%$, the relative error of other forecasting models [4].

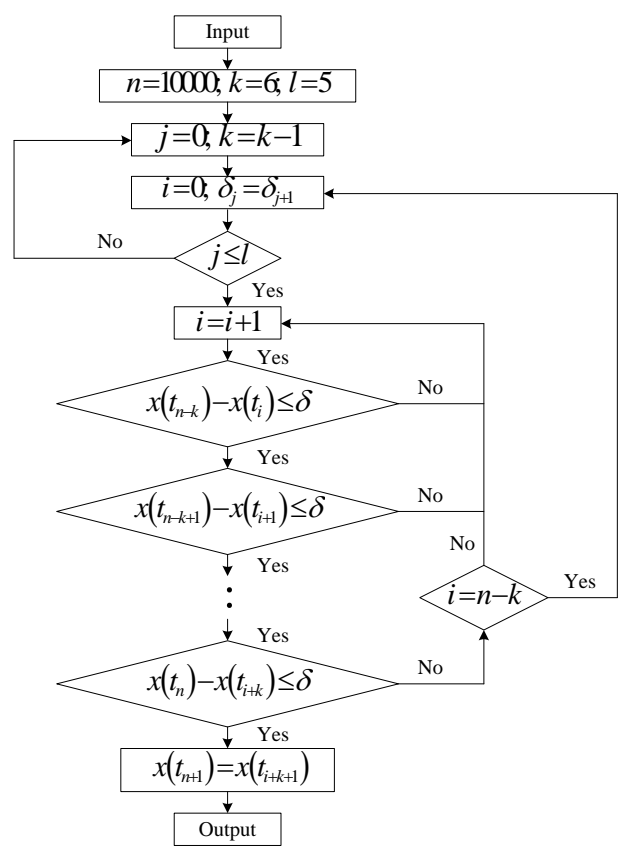

Figure 4. Flow chart of local comparison method

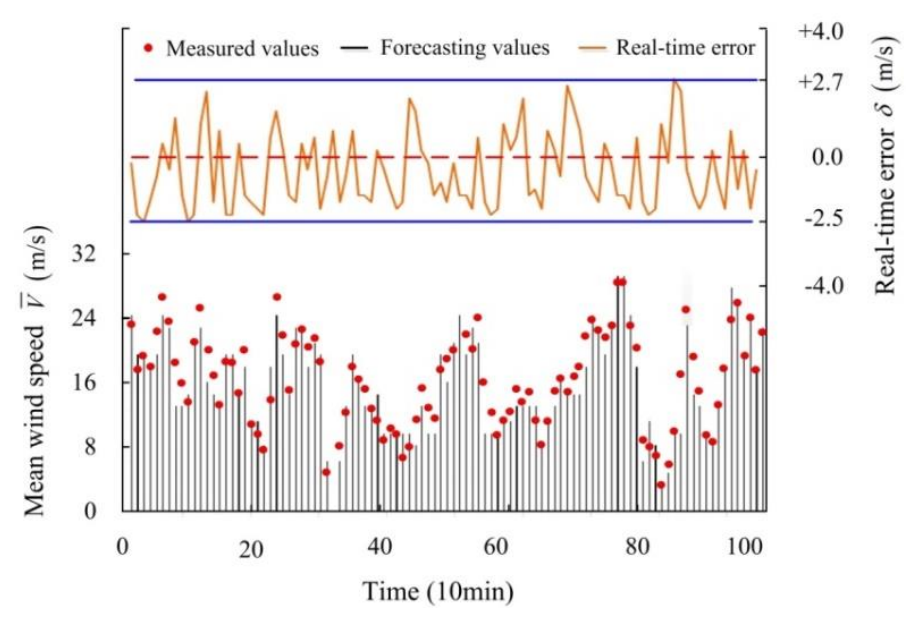

Figure 5. Forecasting results of mean wind speed

The mean wind speed is forecasted by local comparison, but it is still not enough to ensure the safety of wind turbine blades. The possibility of extreme wind speeds that may lead to safety accidents should also be concerned [14]. According to the definition of multi-state random variables, random wind speed can be regarded as a multi-state random variable. Statistically, it is in a certain state at any time with a certain probability. The probabilities of the risk states are exactly what we need to pay attention to. By UGF, the state probability allocation and risk state probability are introduced in Section 3.

\section{State Probability Allocation}

\subsection{Multi-State Random Variable and UGF}

Random wind speed can be regarded as a multi-state random variable, which will be in multiple states with different probabilities at the same time. The measured wind speed at any time can be regarded as the mean value of the random wind speed at that time. It is supposed that the random wind speed has $m$ states, corresponding to $m$ state probabilities. According to the basic idea of UGF, the state value vector and state probability vector of wind speed at time $t$ are given as $\mathbf{v}=\left\{v_{1}, v_{2}, \cdots, v_{m}\right\}$ and $\mathbf{p}(t)=\left\{p_{1}(t), p_{2}(t), \cdots, p_{m}(t)\right\}$. The UGF of $V(t)$ is [15].

$$
u_{V}(t)=\sum_{i=1}^{m} p_{i}(t) z^{v_{i}}=p_{1}(t) z^{v_{1}}+p_{2}(t) z^{v_{2}}+\cdots+p_{m}(t) z^{v_{m}}
$$

The mean value of $V(t)$ is [15]

$$
\bar{V}(t)=\sum_{i=1}^{m} p_{i}(t) v_{i}=p_{1}(t) v_{1}+p_{2}(t) v_{2}+\cdots+p_{m}(t) v_{m}
$$


By UGF, the mean value (measured value) of the random wind speed at any time is known, but the probability of each state is unknown. The probability of each state counted by historical data cannot reflect the real-time probability, so a probability allocation method is adopted to describe it. It is based on the fact that if a state is closer to the mean wind speed, it is more likely to happen [16].

\subsection{State Probability Allocation}

A rectangular coordinate system is established in Figure 6 . The mean wind speed $v(t)$ and the state values $v_{i}(i=1, \cdots, m)$ are listed on the horizontal axis. The measured wind speed can be regarded as the mean wind speed. The probabilities of the states that are close to the mean wind speed are higher. For example, when the mean wind speed is $5 \mathrm{~m} / \mathrm{s}$, the probability of $4 \mathrm{~m} / \mathrm{s}$ is higher than that of $10 \mathrm{~m} / \mathrm{s}$. At present, the relationship between state probability and distance is not accurate. Thus, it is assumed to be linear, and more appropriate relationships than linear may be found in later studies. The difference between $v(t)$ and $v_{i}$ is

$$
\Delta v_{i}=\left|v(t)-v_{i}\right|, i=1,2, \cdots, m
$$

Where $\triangle v_{i}$ denotes the difference between $v(t)$ and $v_{i}$, and $v_{i}$ denotes the $i^{\text {th }}$ state value of the wind speed.

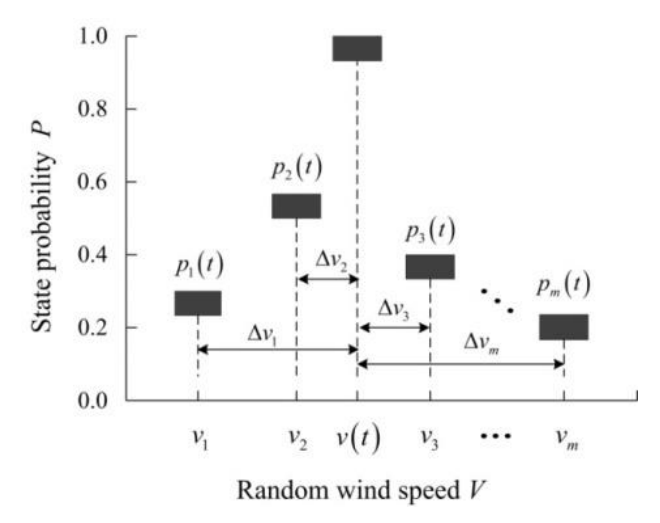

Figure 6. Schematic diagram of state probability allocation

It is supposed that the probability of each state is inversely proportional to its distance to the mean wind speed. Thus, the probability of $v_{i}$ at time $t$ is

$$
p_{i}^{\prime}(t)=\frac{\sum_{j=1}^{m} \Delta v_{j}}{\Delta v_{i}}
$$

Where $p_{i}^{\prime}(t)$ denotes the probability of $v_{i}$ at time $t$.

The sum of the state probabilities is not 1, and the state probabilities need to be standardized in Equation (5).

$$
p_{i}(t)=\frac{p_{i}^{\prime}(t)}{\sum_{j=1}^{m} p_{j}^{\prime}(t)}
$$

Where $p_{i}(t)$ denotes the probability of $v_{i}$ after standardization.

Therefore, the UGF of $v(t)$ is expressed as [15] 


$$
u_{V}(z, t)=\sum_{i=1}^{m} p_{i}(t) z^{v_{i}}=p_{1}(t) z^{v_{1}}+p_{2}(t) z^{v_{2}}+\cdots+p_{m}(t) z^{v_{m}}
$$

If the state $v_{k}(k=1, \cdots, m)$ is a risk state and the probability of the risk state $v_{k}$ at time $t$ is $p_{k}(t)$, the UGF of $v_{k}$ is expressed as $p_{k}(t) z^{v_{k}}[15]$.

\subsection{State Probability Matrix}

In order to store the state probability of each time conveniently, a state probability matrix is established in Equation (7). The time is expressed in the column of the matrix, and the state values of the wind speed are expressed in the row of the matrix. The state probabilities of each time from 1 to $t$ are listed on the left side of the vertical line. According to Equations (3) to (6), the forecasted value of $v_{f}(t+1)$ can be forecasted by local comparison in Figure 4. The state probabilities allocated according to the forecasting value of $v_{f}(t+1)$ are listed on the right side.

$$
\begin{aligned}
& \begin{array}{lllll}
1 & 2 & \ldots & t & t+1
\end{array} \\
& \begin{array}{c}
v_{1} \\
v_{2} \\
\vdots \\
v_{m}
\end{array}\left[\begin{array}{cccc|c}
p_{1}(1) & p_{1}(2) & \cdots & p_{1}(t) & p_{1}(t+1) \\
p_{2}(1) & p_{2}(2) & \cdots & p_{2}(t) & p_{2}(t+1) \\
\vdots & \vdots & \ddots & \vdots & \vdots \\
p_{m}(1) & p_{m}(2) & \cdots & p_{m}(t) & p_{m}(t+1)
\end{array}\right]
\end{aligned}
$$

In order to ensure sufficient computing speed, according to the idea of equal dimension filling [17], when $t=t+1$, the probabilities at time 1 are deleted. The forecasted wind speed $v_{f}(t+1)$ is replaced by its measured value $v_{a}(t+1)$, and the probabilities at $t+1$ are allocated according the measured value. Thus, the state probabilities of each time from 2 to $t+1$ are listed on the left side of the vertical line. According to Equations (3) to (6), the forecasting value of $v_{f}(t+2)$ can be forecasted by local comparison in Figure 4 . The state probabilities allocated according to the forecasting value of $v_{f}(t+2)$ are listed on the right side. The probabilities at $t+3, t+4$, and so on will be obtained in the same manner.

\subsection{Statistics of Risk State Probabilities}

The main purpose of the state probability matrix is to identify the risk state probabilities and provide services for the prognostic and health management (PHM) of wind turbine blades. Thus, after obtaining the state probability matrix at each time, the risk state probability curve can be drawn by picking out the risk state probabilities. 100 risk state probabilities are selected, and the risk state probability curve is shown in Figure 7. The risk states mentioned here may be more than one state. For example, if $15 \mathrm{~m} / \mathrm{s}$ is the acceptable maximum wind speed, $16 \mathrm{~m} / \mathrm{s}, 17 \mathrm{~m} / \mathrm{s}$, and so on are all risk states. In this case, the risk state includes the wind speeds that are greater than $22 \mathrm{~m} / \mathrm{s}$ (the warning wind speed). With $p_{k}(t)$, the probability of the risk state $v_{k}(k=1, \cdots, m)$, the risk wind load of the wind turbine blade is forecasted, the safety condition of the wind turbine blade can be conveniently observed, the information basis for the operation control of the wind turbine blade is provided, and the dangerous situation of the wind turbine blade may be avoided as early as possible.

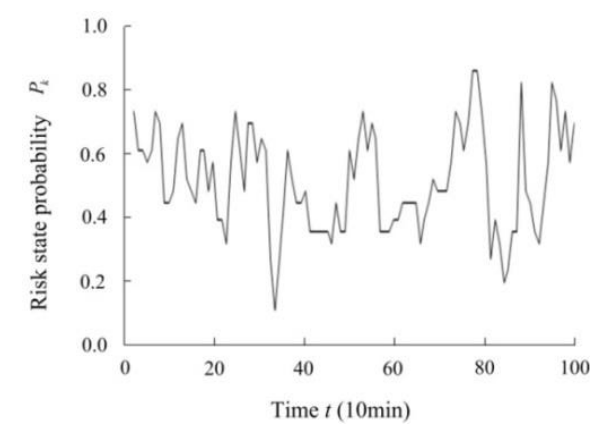

Figure 7. Statistics of risk state probability 


\section{Maximum Wind Speed Forecasting}

The average working condition of the wind turbine blade in ten minutes is described by the mean wind speed and risk state probability. However, the wind speed in each ten-minute interval is not always near the average wind speed, and sudden changes often appear at some certain time points. If the maximum wind speed within ten minutes can be forecasted, the worst situation that the wind turbine blade will face can be found in advance. In conclusion, it is necessary to monitor and forecast the maximum wind speed in ten minutes. When the local comparison is planned to forecast the maximum wind speed, the forecasting error is large and the results are scattered. This may be due to the strong randomness of the maximum wind speed. Some other methods are needed to forecast the maximum wind speed. With the same data resources as Figure 2 , the difference between the maximum wind speed and mean wind speed is calculated, and the product of the risk state value and risk state probability is calculated. Their ratio is expressed as

$$
\tau(t)=\frac{v_{m}(t)-\bar{v}(t)}{v_{k} \times p_{k}(t)}
$$

where $v_{m}(t)$ denotes the maximum wind speed, $\bar{v}(t)$ denotes the mean wind speed, and $\tau(t)$ denotes the ratio.

The calculation result is that $\tau(t)$ tends to a constant $\tau$. In this case, $\tau$ is about $0.73 . \tau$ may be different in other wind speed data. It is not difficult to understand that when the average wind speed is large, it is closer to the risk state, so the risk state probability is large. Therefore, a relationship among the mean wind speed, risk state probability and maximum wind speed is

$$
v_{m}(t)=\bar{v}(t)+\tau \times v_{k} \times p_{k}(t)
$$

Using Equation (9), the maximum wind speed can be forecasted indirectly. The calculation results of the maximum wind speed and the real-time errors between the measured values and forecasting values are shown in Figure 8 . The errors are between -3.1 and +2.9 , and the maximum of relative error is about $15 \%$, which is less than $17 \%$. The accuracy of the maximum wind speed forecasting is higher, which shows that state probability allocation based on the mean wind speed is reasonable.

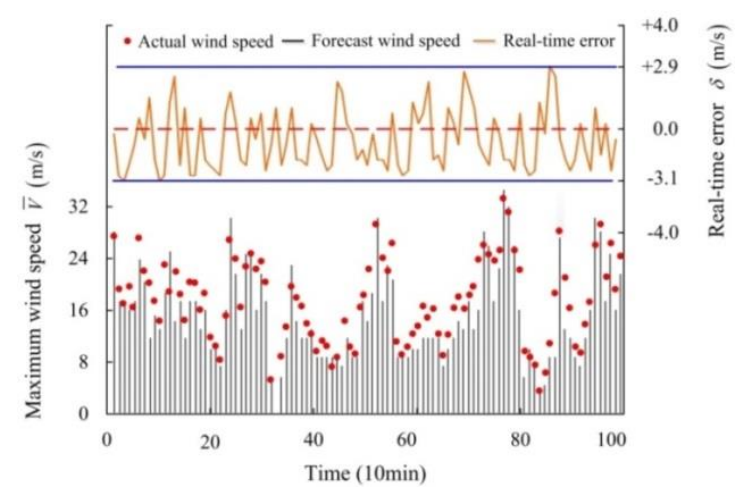

Figure 8. Forecasting results of maximum wind speed

\section{Error Forecasting and Wind Speed Correction}

It is impossible to forecast wind speed without any error. If the error can be forecasted, the forecasting wind speed may be corrected to be more accurate. Here, local comparison is used to forecast the error for one time. The one-time forecasting error is used to correct the forecasted wind speed for one time. After one-time correction, the one-time forecasting error is forecasted for two times, and the two-time forecasting error is used to correct the one-time corrected wind speed. Error forecasting and wind speed correction are carried out many times. The specific steps are as follows:

Step 1 The forecasting values of several errors are obtained, which are called one-time forecasting errors. The one-time forecasting errors are used to correct the wind speeds for one time, which are called one-time corrected wind speeds. 
Step 2 According to the error data of one-time corrected wind speeds, using local comparison, the forecasting values of several one-time forecasting errors are obtained, which are called two-time forecasting errors. The two-time forecasting errors are used to correct the one-time corrected wind speeds, which are called two-time corrected wind speeds.

Step 3 In the same way, the three-time forecasting errors are obtained and the two-time corrected wind speeds are corrected. This continues for four-time, five-time, and so on. After three-time and four-time correction, the forecasting errors are almost unchanged and the effect of wind speed correction is negligible. Thus, one-time and two-time correction are carried out in this paper.

Forecasting errors of mean wind speeds before and after correction are shown in Figure 9. Before correction, the forecasting errors are between -2.5 and +2.7 . After one-time correction, the one-time forecasting errors are between -1.3 and +1.4. After two-time correction, the two-time forecasting errors are between -1.0 and +1.1 . The relative error after two-time correction is about $7 \%$, which is far smaller than $17 \%$. Forecasting errors of maximum wind speeds before and after correction are shown in Figure 10. Before correction, the forecasting error is between -3.1 and +2.9 . After one-time correction, the one-time forecasting errors are between -1.9 and +1.6 . After two-time correction, the two-time forecasting errors are between -1.4 and +1.2 . The relative error after two-time correction is about $10 \%$, which is far smaller than $17 \%$.

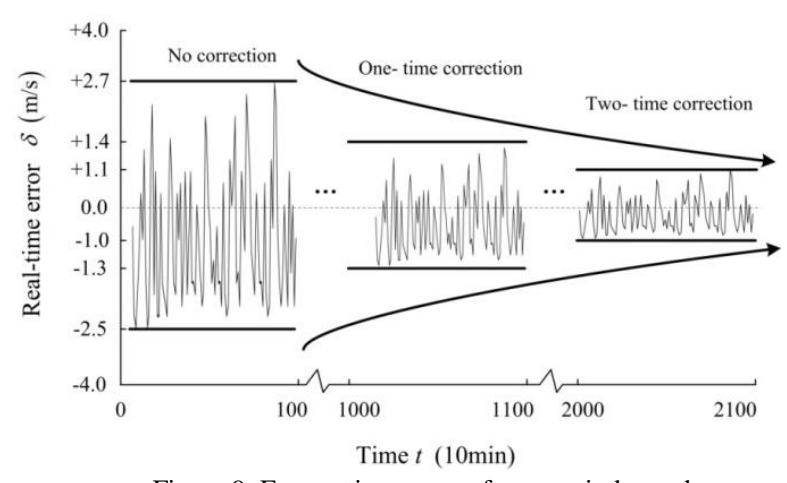

Figure 9. Forecasting errors of mean wind speed

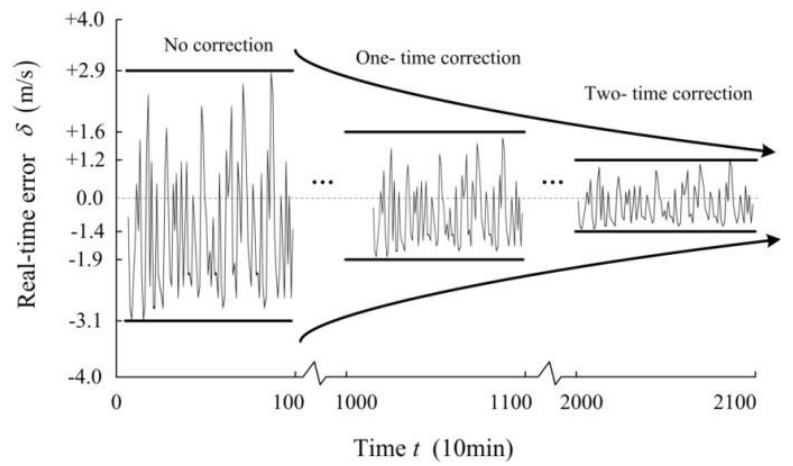

Figure 10. Forecasting errors of maximum wind speed

From Figures 9 and 10, the forecasting error can be significantly reduced through error forecasting and wind speed correction.

\section{Average Relative Error}

Once the forecasting model of wind speed is established, the forecasting error is inevitable. The forecasting error is related to the error accumulation of the forecasting model during many times of forecasting. Therefore, the average relative error should be counted to characterize the cumulative error of the model. The average relative error of the mean wind speed is taken as the average relative error of the forecasting model. The average relative error at time $t$ is defined as

$$
\overline{\delta_{r}(t)}=\frac{1}{t-1} \sum_{j=1}^{t-1}\left|\frac{\delta(j)}{\overline{v_{a}}(j)}\right|
$$

where $\overline{\delta_{r}(t)}$ denotes the average relative error at time $t, \delta(j)$ denotes the real-time error at time $j$, and $\overline{v_{a}}(j)$ denotes the measured value of the mean wind speed at time $j$.

The real-time error $\delta(j)$ is expressed as

$$
\delta(j)=\overline{v_{f}}(j)-\overline{v_{a}}(j)
$$

where $\overline{v_{f}}(j)$ denotes the forecasting value of the wind speed at time $j$.

According to Equations (10) and (11), the average relative errors of the mean wind speeds before and after correction 
are shown in Figure 11. Before wind speed correction, the average errors are between $9 \%$ and $15 \%$; by one-time wind speed correction, they are between $6 \%$ and $11 \%$; and by two-time wind speed correction, they are between $3 \%$ and $7 \%$. In each of the three stages, due to error accumulation, the average relative errors change from small to large. The average relative error is a reflection of the model error level, in other words, the reflection of the credibility of wind speed forecasting.

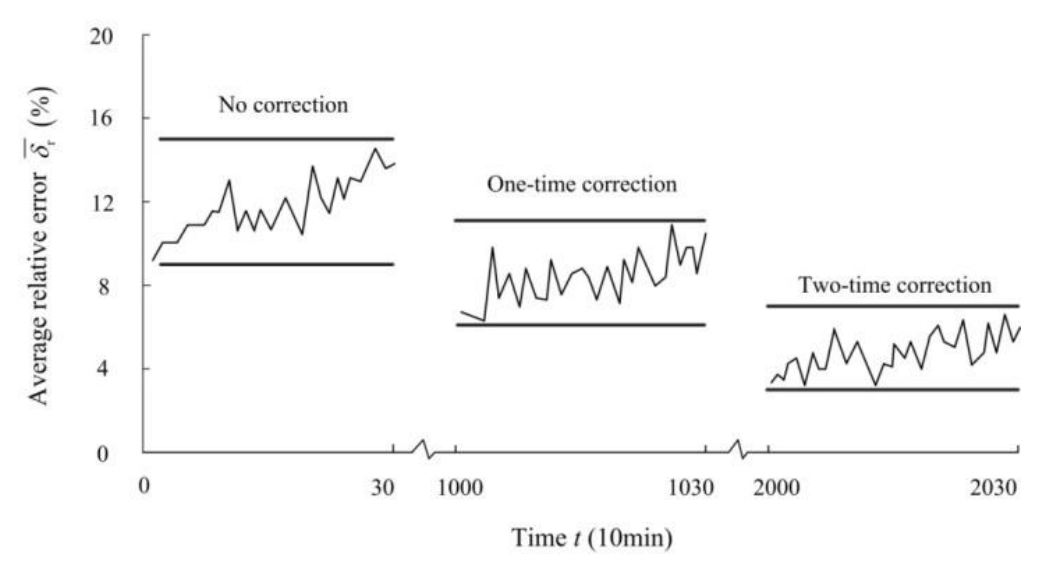

Figure 11. Average relative errors before and after correction

\section{Mean Circular Tube Model}

\subsection{Mean Circle}

Random wind speed has been regarded as the multi-state random variable in Section 3. The state values and their probabilities of wind speed $V(t)$ are represented by line segments with arrows. The sizes of state values are represented by the lengths of line segments, they are arranged around the point $O$ clockwise from small to large. By taking $O$ as the center and taking the mean value of $V(t)$ as the radius, the mean circle is built in Figure 12. The most outlying green area is called the buffer area. The width of the buffer area is

$$
\Delta v=v_{r}-v_{n}
$$

where $\Delta v$ denotes the width of the buffer area, $v_{n}$ denotes the warning wind speed, and $v_{r}$ denotes the critical wind speed.

On location $\mathrm{B}$, the wind turbine blade is under critical wind speed with danger. On location A, the wind is between $v_{n}$ and $v_{r}$, and the wind turbine blade is unhealthy. $v_{n}$ can be determined by observing the normal fluctuation range of the wind speed, and $v_{r}$ can be determined by viewing the output power of the wind turbine. It is expressed as

$$
\left\{\begin{array}{l}
v(t)<v_{r}, P_{\text {out }}(t)>0 \\
v(t) \geq v_{r}, P_{\text {out }}(t)=0
\end{array}\right.
$$

where $v$ denotes the measured wind speed at time $t$ and $P_{\text {out }}(t)$ denotes the output power of the wind turbine at time $t$.

The $2.5 \mathrm{MW}$ wind turbine is taken as an example, the cut-in wind speed is $3 \mathrm{~m} / \mathrm{s}$, and the rated wind speed is $15 \mathrm{~m} / \mathrm{s}$. From Figure 2, the wind speed fluctuates steadily from $3 \mathrm{~m} / \mathrm{s}$ to $15 \mathrm{~m} / \mathrm{s}$, so $v_{\mathrm{n}}$ is determined to be $15 \mathrm{~m} / \mathrm{s}$. The cut-out wind speed of the wind turbine is $22 \mathrm{~m} / \mathrm{s}$, and the records shows that $P_{\text {out }}(t)$ is zero when $v$ is higher than $22 \mathrm{~m} / \mathrm{s}$. Thus, $v_{r}$ is determined to be $22 \mathrm{~m} / \mathrm{s}$.

\subsection{Mean Circular Tube}

A plane coordinate system is established in Figure 13. The mean circles at every moment are listed in the horizontal axis. A series of buffer areas is superimposed as a circular tube, which is called the mean circular tube. 


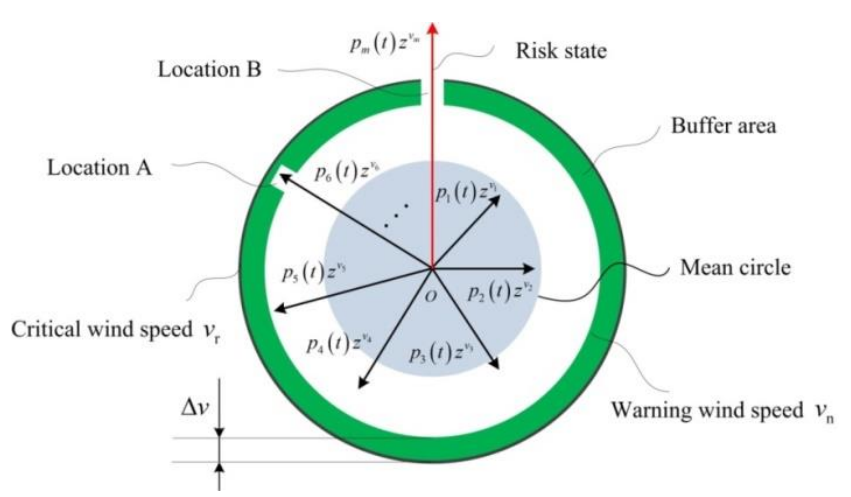

Figure 12. Schematic diagram of mean circle

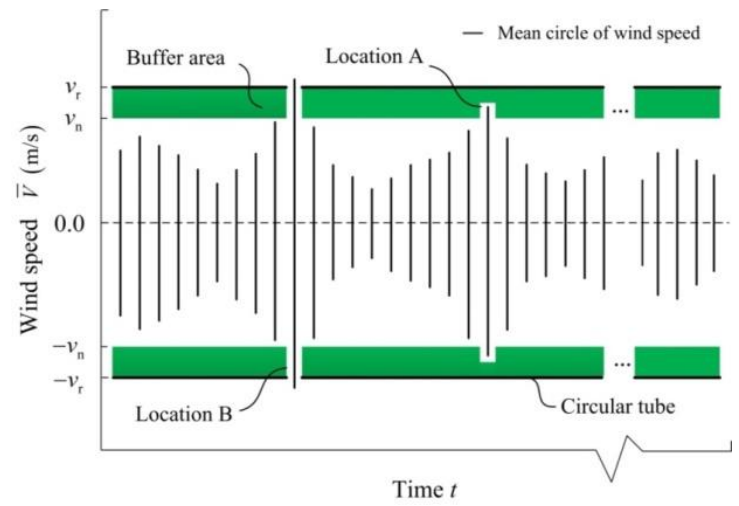

Figure 13. Schematic diagram of mean circular tube

\subsection{Combined Mean Circular Tube}

In Figure 14, a combined model should be established to monitor the average wind speed, maximum wind speed, risk state probability, and average relative error centrally. They are marked by four independent coordinates in the same plane.

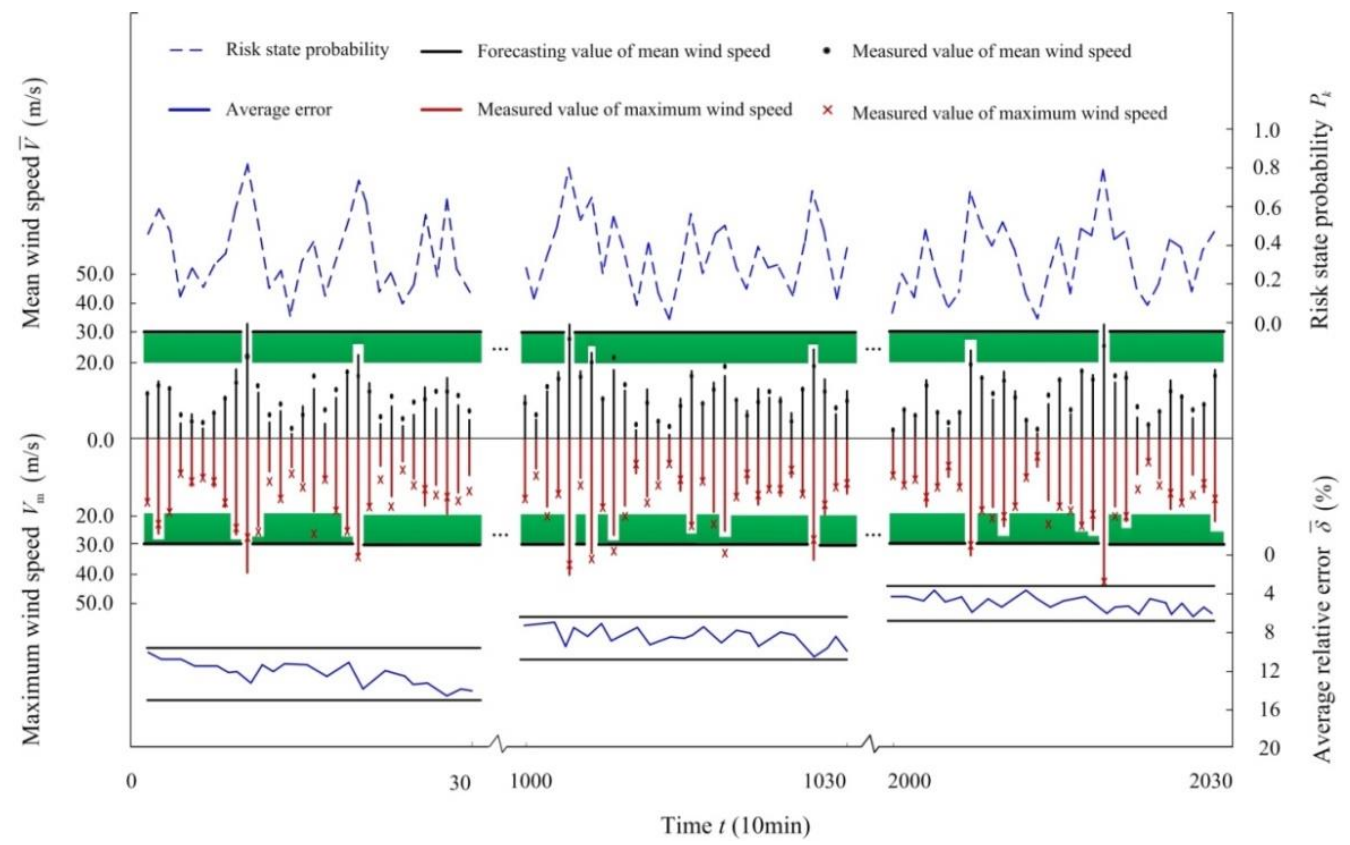

Figure 14. Combined mean circular tube of wind speed

From Figure 14, it can be easily observed in real time whether the wind speeds enter the buffer or break through the circular tube, whether it is possible for the risk states to happen and the probabilities, and whether the forecasting results are credible and how about the credibilities of them. The discovery of the law of wind speeds is to master the law of loads, which is very important for the condition monitoring and forecasting of wind turbine blades.

\section{Conclusions}

In this paper, the three specific works are as follows:

(1) Based on the periodicity, repeatability, and trends of wind speed, the local comparison method is established to forecast the mean wind speed. The state probability allocation method is established to obtain the risk state probability, and then the maximum wind speed is calculated.

(2) The forecasting errors are forecasted twice using the local comparison method, and the wind speeds are corrected by the two-time forecasting error, which makes the forecasted wind speeds much closer to their measured values. 
(3) By establishing the mean circular tube model, the mean wind speed, maximum wind speed, risk state probability, and average relative error are monitored centrally, which makes the operation and control of wind turbine blades more intuitive.

Based on the above three works, the two main conclusions are as follows:

(1) Using the local comparison method and state probability allocation method to forecast the wind speed, the forecasting error of the mean wind speed is between -2.5 and +2.7 , and the forecasting error of the maximum wind speed is between -3.1 and +2.9 . The relative error of the mean wind speed forecasting is less than $13 \%$, and the relative error of the maximum wind speed forecasting is less than $15 \%$. Both are smaller than $17 \%$, the relative error of the other wind speed forecasting model.

(2) After two-time error forecasting and two-time wind speed correction, the forecasting error of the mean wind speed is between -1.0 and +1.1 , and the forecasting error of the maximum wind speed is between -1.4 and +1.2 . The relative error of the mean wind speed forecasting is less than $7 \%$, and the relative error of the maximum wind speed forecasting is less than $10 \%$. Error forecasting and wind speed correction are effective for improving the forecasting accuracy of wind speed.

\section{Acknowledgments}

This research was supported by the National Natural Science Foundation of China (No. 51665029).

\section{References}

1. B. Yang and D. Sun, "Testing, Inspecting and Monitoring Technologies for Wind Turbine Blades: A Survey," Renewable \& Sustainable Energy Reviews, Vol. 22, No. 8, pp. 515-526, June 2013

2. D. He, E. Bechhoefer, and A. Saxena, "Special Issue on Wind Turbine Prognostics and Health Management," Phmsociety Org, 2013

3. W. P. Mahoney, K. Parks, G. Wiener, Y. Liu, W. L. Myers, J. Sun, et al., "A Wind Power Forecasting System to Optimize Grid Integration," IEEE Transactions on Sustainable Energy, Vol. 3, No. 4, pp. 670-682, October 2012

4. Y. Ren, P. N. Suganthan, and N. Srikanth, "A Comparative Study of Empirical Mode Decomposition-based Short-Term Wind Speed Forecasting Methods," IEEE Transactions on Sustainable Energy, Vol. 6, No. 1, pp. 236-244, 2017

5. J. J. Yun, W. C. Chan, J. H. Lee, and K. W. Kang, "Development of Fatigue Life Prediction Method and Effect of 10-Minute Mean Wind Speed Distribution on Fatigue Life of Small Wind Turbine Composite Blade," Renewable Energy, Vol. 79, No. 1, pp. 187-198, July 2014

6. S. Dutta, Y. Li, A. Venkataraman, L. M. Costa, T. Jiang, R. Plana, et al., "Load and Renewable Energy Forecasting for a Microgrid using Persistence Technique," Energy Procedia, Vol. 143, pp. 617-622, December 2017

7. R. Ak, O. Fink, and E. Zio, "Two Machine Learning Approaches for Short-Term Wind Speed Time-Series Prediction," IEEE Transactions on Neural Networks \& Learning Systems, Vol. 27, No. 8, pp. 1734-1747, August 2016

8. H. Quan, D. Srinivasan, and A. Khosravi, "Incorporating Wind Power Forecast Uncertainties into Stochastic Unit Commitment using Neural Network-based Prediction Intervals," IEEE Transactions on Neural Networks \& Learning Systems, Vol. 26, No. 9, pp. 2123-2135, September 2015

9. J. Y. Lu, Y. X. Peng, M. Wang, S. J. Gu, and M. X. Zhao, "Support Vector Machine Combined with Distance Correlation Learning for Dst, Forecasting During Intense Geomagnetic Storms," Planetary \& Space Science, Vol. 120, pp. 48-55, January 2016

10. H. Takeda, Y. Tamura, and S. Sato, "Using the Ensemble Kalman Filter for Electricity Load Forecasting and Analysis," Energy, Vol. 104, pp. 184-198, June 2016

11. X. Jiang, C. Jiang, M. Peng, and H. Lin, “A Short-Term Combination Wind Speed Forecasting Method Considering Seasonal Periodicity and Time-Continuity," Automation of Electric Power Systems, Vol. 34, No. 15, pp. 75-79, August 2010

12. Y. A. Fetisova, B. V. Ermolenko, G. V. Ermolenko, and S. V. Kiseleva, "Determining the Parameters of Weibull Function to Estimate the Wind Power Potential in Conditions of Limited Source Meteorological Data," Thermal Engineering, Vol. 64, No. 4, pp. 251-257, April 2017

13. M. Laapas and A. Venäläinen, "Homogenization and Trend Analysis of Monthly Mean and Maximum Wind Speed Time Series in Finland, 1959-2015," International Journal of Climatology, No. 10, May 2017

14. Y. Q. Xiao, Q. S. Li, Z. N. Li, Y. W. Chowa, and G. Q. Lic, "Probability Distributions of Extreme Wind Speed and its Occurrence Interval," Engineering Structures, Vol. 28, No. 8, pp. 1173-1181, July 2006

15. Z. W. An, H. Z. Huang, and L. Yu, "A Discrete Stress-Strength Interference Model based on Universal Generating Function," Reliability Engineering \& System Safety, Vol. 93, No. 10, pp. 1485-1490, October 2008

16. T. Jiang and Y. Liu, "Parameter Inference for Non-Repairable Multi-State System Reliability Models by Multi-Level Observation Sequences," Reliability Engineering \& System Safety, Vol. 166, pp. 3-15, November 2016

17. Z. Anbing, "A Prediction Boundary Determination Method of the Equal-Dimension Gray Filling Model based on Law of Error Propagation," Science of Surveying \& Mapping, October 2017 
Xuezong Bai is a Ph.D. student in the School of Mechatronics Engineering at Lanzhou University of Technology, China. His research interests include the mechanical prognostic and health management (PHM) of mechanical equipment and components.

Zongwen An is a professor at Lanzhou University of Technology. He received his Ph.D. in mechanical engineering from the University of Electronic Science and Technology of China. He is a senior member of the Chinese Mechanical Engineering Society. His research interests include structural reliability and mechanical design theory.

Yunfeng Hou is a professor at Lanzhou University of Technology. He received his Ph.D. from Lanzhou University of Technology. His research interests include special equipment and its control, modern design methods and theory, and superfine grinding technology.

Jianxiong Gao is a Ph.D. student in the School of Mechatronics Engineering at Lanzhou University of Technology. His research interests include mechanical strength theory and structural reliability. 